ORIGINAL ARTICLE

\title{
Severe psychosocial deprivation in early childhood is associated with increased DNA methylation across a region spanning the transcription start site of $C Y P 2 E 1$
}

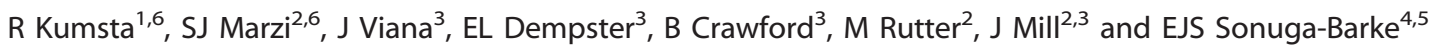

Exposure to adverse rearing environments including institutional deprivation and severe childhood abuse is associated with an increased risk for mental and physical health problems across the lifespan. Although the mechanisms mediating these effects are not known, recent work in rodent models suggests that epigenetic processes may be involved. We studied the impact of severe early-life adversity on epigenetic variation in a sample of adolescents adopted from the severely depriving orphanages of the Romanian communist era in the 1980s. We quantified buccal cell DNA methylation at $\sim 400000$ sites across the genome in Romanian adoptees exposed to either extended (6-43 months; $n=16$ ) or limited duration ( $<6$ months; $n=17$ ) of severe early-life deprivation, in addition to a matched sample of UK adoptees $(n=16)$ not exposed to severe deprivation. Although no probe-wise differences remained significant after controlling for the number of probes tested, we identified an exposure-associated differentially methylated region (DMR) spanning nine sequential CpG sites in the promoter-regulatory region of the cytochrome P450 2E1 gene (CYP2E1) on chromosome 10 (corrected $P=2.98 \times 10^{-5}$ ). Elevated DNA methylation across this region was also associated with deprivation-related clinical markers of impaired social cognition. Our data suggest that environmental insults of sufficient biological impact during early development are associated with long-lasting epigenetic changes, potentially reflecting a biological mechanism linking the effects of early-life adversity to cognitive and neurobiological phenotypes.

Translational Psychiatry (2016) 6, e830; doi:10.1038/tp.2016.95; published online 7 June 2016

\section{INTRODUCTION}

Brain circuits underpinning cognition and socioemotional functioning are sculpted by social experiences during early life. ${ }^{1}$ Deficient or adverse social environments during this period can increase long-term vulnerability for psychiatric disorders. ${ }^{2}$ Understanding the mechanisms linking negative experiences to chronic mental health effects is a key target for translational developmental neurobiology. ${ }^{3}$ One hypothesis is that severe social adversity induces long-term alterations to gene expression and function through dynamic epigenetic modifications. ${ }^{4}$ Experimental studies in model organisms, for example, have shown that variation in maternal behavior brings about epigenetic alterations and associated changes in gene expression at specific loci, underlying life-long phenotypic differences in physiology and behavior, including neuroendocrine stress responsiveness, fearrelated behavior and attentional processes, cognitive development, female reproductive behavior and maternal care itself (see Zhang and Meaney ${ }^{5}$ for a review). There is some evidence for similar epigenetic alterations in response to various environmental stressors in humans, including prenatal exposure to famine, ${ }^{6}$ psychosocial stress during infancy and pre-school years, ${ }^{7}$ early-life socioeconomic status, ${ }^{8}$ and childhood abuse. ${ }^{4,9-11}$ However, direct replication of the effects observed in experimental animal models in humans remains challenging for a number of reasons. Most sample cohorts are characterised by considerable heterogeneity in the nature, timing and severity of adverse exposures, and there is considerable confounding between early and continuing later adversity and between adversity and consequent mental health problems.

Because of necessary ethical constraints, 'natural experiments' - that is, studies in which exposure to severe adversity is not under direct experimental control-are the best available method for examining epigenetic changes following exposure to severe environmental conditions in human populations. ${ }^{12}$ The English and Romanian Adoptees study (ERA) is a prospective longitudinal study of the effects of severe adversity experienced by children before the age of 43 months in grossly depriving Romanian orphanages before they were adopted into UK families at the fall of the Ceauşescu regime in $1989 .^{13}$ The children were followed across development and have been assessed at ages 4, 6, 11 and 15 years, with follow-up data collection at the age of 23 years just completed. ERA represents a powerful 'natural experiment' to test the epigenetic hypothesis of the effects of psychosocial adversity. This is because the ERA children (i) were typically exposed to severe deprivation from just after birth for variable, but defined, periods of time ( 2 weeks to 43 months) and (ii) then experienced a sudden, precisely timed, radical change from a profoundly depriving environment to a nurturing adoptive

\footnotetext{
${ }^{1}$ Department of Genetic Psychology, Faculty of Psychology, Ruhr-University Bochum, Bochum, Germany; ${ }^{2}$ MRC Social, Genetic and Developmental Psychiatry Centre, Institute of Psychiatry, Psychology and Neuroscience, King's College London, London, UK; ${ }^{3}$ University of Exeter Medical School, University of Exeter, Exeter, UK; ${ }^{4}$ Department of Psychology, Institute for Disorders of Impulse and Attention, Developmental Brain-Behavior Laboratory, University of Southampton, Southampton, UK and ${ }^{5}$ Department of Experimental

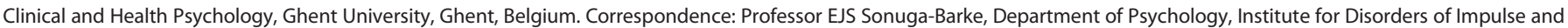
Attention, Developmental Brain-Behaviour Laboratory, University of Southampton, Building 44, Highfield Campus, Southampton SO17 1 BJ, UK.

E-mail: ejb3@soton.ac.uk
}

${ }^{6}$ These authors contributed equally to this work.

Received 14 January 2016; revised 22 March 2016; accepted 31 March 2016 
family one. Furthermore, whereas many in the cohort have displayed long-term persistent deprivation-related problems, at least to adolescence, other adoptees are highly resilient, being indistinguishable in terms of mental health compared with their non-deprived peers. There is a strong association between length of institutional deprivation and risk for persisting deficits. Individuals adopted before 6 months were found to have rates of impairment no different from non-exposed populations, whereas about half of the samples adopted between the ages of 6 and 43 months showed continuing psychological deficits to adolescence. ${ }^{14}$ Within the ERA cohort, early adversity is associated with both intellectual and social behavioral deficits, with a characteristic pattern of social impairment across two domains. The first has been termed quasi-autism and is a behavioral pattern characterized by autistic-like features, particularly abnormal preoccupations and intense circumscribed interests. The difference to classical autism lies in greater, albeit unusual, social interest and flexibility, and in the diminishing intensity of these features over time. ${ }^{15}$ Deficits in Theory of Mind (ToM) provide substantial mediation of the quasi-autistic pattern. ${ }^{16,17}$ The second shares many features with the new DSM diagnostic category 'Disinhibited Social Engagement Disorder'18 and is characterized by a marked disregard for social boundaries, inappropriate levels of familiarity, social disinhibition and self-disclosure. ${ }^{15,19}$ Across all ages there is a substantial overlap between quasi-autism and disinhibited social engagement. ${ }^{15}$ These core deficits in social cognition and behavior are often accompanied by cognitive deficits (at the age of 15, the mean intelligence quotient (IQ) of the late adopted group was one s.d. below the UK and early adopted group) and symptoms of attention-deficit hyperactivity disorder. ${ }^{20}$

The persistent nature of the negative impact of early severe deprivation, which for many in the sample was not eradicated by positive post-adoption experiences, is consistent with an enduring biological impact of early deprivation. In this study we aimed to test whether exposure to extreme deprivation is associated with altered DNA methylation among Romanian adoptees and whether these effects are also related to variation in intellectual and social functioning in the ERA group. ${ }^{17}$

\section{MATERIALS AND METHODS}

\section{Sample}

The ERA sample was drawn from children adopted from Romania into families residing in England between February 1990 and September 1992, who were aged 43 months, or below, at the time of entry to the United Kingdom (see Rutter et al. ${ }^{13}$ for detailed description of historical background and sample characteristics). Briefly, following an agestratified sampling design, the ERA study enrolled roughly equal numbers of children adopted before 6 months, between 6 and 24 months and over 24-43 months. The Romanian children were compared with a group of 52 children born and adopted within the United Kingdom before the age of 6 months. None of the children in the within-UK adoptee group had been exposed to early deprivation, neglect or abuse. Most children had been placed in institutions in the first weeks of life (the mean age of entry was 0.34 months, s.d. $=1.26$, making it unlikely that the reason for their admission into institutions was manifest handicap). Out of 217 subjects, DNA samples were available for 131 individuals, and 49 individuals were selected for the present study. Our selection strategy was based on the finding that at 11 and 15 years of follow-up there was a step-wise relationship between length of institutional rearing and risk for psychosocial and developmental outcome, with the difference laying between institutional deprivation that did not continue beyond the age of 6 months and institutional deprivation that persisted longer than that. ${ }^{13}$ Accordingly, for the current analyses we focused on the comparison between individuals experiencing extended (more than 6 months; $n=16$ ) or limited (less than 6 months; $n=17$ ) deprivation. Furthermore, these two groups were compared with a subgroup of individuals from the within-UK adoptee group $(n=16)$. Selection of the participants was carried out at random for the UK comparison group and the group experiencing $<6$-month deprivation. For the $>6$-month category, participants were selected at random from the subgroup of individuals showing deprivationrelated impairments. As shown in Supplementary Table 1 and Supplementary Table 2, there were no significant differences between groups in gender, smoking or the abuse of alcohol, cannabis or other drugs. With the exception of one individual in the $>6$-month exposure group using antidepressants at time of sampling, and the elevated rate of methylphenidate use in the $>6$-month exposure group, there was no use of medication among the samples included in this study (antipsychotics, mood stabilizers and antidepressants). Furthermore, there were no significant differences in birth weight between the early and late adopted Romanian adoptees. As shown in Supplementary Table 1, and comparable to the ERA sample as a whole, ${ }^{16}$ deficits in ToM and lower IQ were observed in the group with extended deprivation. Furthermore, as previously observed, ${ }_{13}^{13}$ there were no differences between the short length of deprivation and the UK comparison group. The study was approved by the King's College London ethics committee. Parents gave informed consent for themselves and their children.

\section{DNA methylation profiling}

Buccal cell samples were collected at the age of 15 and DNA isolated using a standard method. ${ }^{21}$ Genomic DNA was treated with sodium bisulfite in duplicate using the EZ-96 DNA methylation-gold kit (Zymo Research, Irvine, CA, USA) and DNA methylation profiled using the Infinium HumanMethylation450 BeadChip (Illumina, San Diego, CA, USA) processed on an Illumina HiScan System (Illumina) according to the manufacturers' standard protocol. All samples were randomized within and between arrays to avoid potential batch effects.

\section{Data-processing and quality control}

Signal intensities for each probe were extracted using the Illumina GenomeStudio software and were imported into R (ref. 22) using the methylumi ${ }^{23}$ and minfi package. ${ }^{24}$ Multidimensional scaling plots of variable probes on the sex chromosomes were used to check that the predicted gender corresponded with the reported gender for each individual. Stringent quality-control checks, quantile normalization and separate background adjustment of methylated and unmethylated intensities of type I and II probes were implemented using the wateRmelon package in . $^{25}$ Samples with $\leqslant 5 \%$ of sites with a detection $P$-value $>0.05$ were included in subsequent analyses, and probes with $>5 \%$ of samples with a detection $P$-value $>0.05$ or a bead count $<3$ in $5 \%$ of samples were removed. We excluded the 65 single-nucleotide polymorphism probes, probes on sex chromosomes, cross-hybridizing probes ${ }^{26}$ and probes with common (minor allele frequency $>5 \%$ ) single-nucleotide polymorphisms in the CG or single-base extension position from subsequent analysis, with the final analysis data set comprising 382291 probes.

\section{Cognitive and sociocognitive abilities}

Cognitive abilities were assessed with the short form of the Wechsler Intelligence Scale for Children (WISC III, UK) at 15 years of follow-up. This is the most commonly used standardized measure of young people's cognitive abilities, and it has established reliability. ${ }^{27}$ Four subscales of the WISC were employed: two from the verbal scales (vocabulary and similarities) and two from the performance scales (block design and object assembly). These four subscales were selected to provide a good estimate of full-scale IQ (reliability coefficient $=0.94$ ). ${ }^{28}$ The four subscales were prorated to form a full-scale IQ for subsequent analyses. At the age of 11 years, the 'strange stories' task ${ }^{29}$ was employed as a measure of ToM. The task required the children to respond to seven ToM-related vignettes. The responses to the vignettes were scored in terms of the level of ToM understanding displayed, with ' 0 ' indicating a non-ToM-related response, ' 1 ' indicating basic-level ToM understanding and ' 2 ' indicating evidence of more sophisticated ToM understanding. Scores were combined across the seven stories, and the mean scores were used in analyses.

\section{Data analysis}

Data was analyzed using a $t$-test for group mean differences in DNA methylation between the two Romanian adoption groups of same ethnicity. No further covariates were included in this test, as all samples were taken at the same age. The potential confounding effect of sex on the identified differences was ruled out through the comparison of results with a sex-regressed model (Supplementary Figure 1). Associations between DNA methylation and exposure time (continuous) as well as IQ and ToM 
were analyzed using linear regression. Region-level analysis for deprivation group, ToM and IQ was performed by spatially combining correlated $P$ values using the Python module comb- . $^{30}$ We allowed a maximum distance of $1000 \mathrm{bp}$ between neighboring CpG sites, and only included probes with a $P$-value $<0.05$ in the initial epigenome wide association scan as starting points for identifying potential differentially methylated regions (DMRs). For each DMR, we report the combined $P$, which is StoufferLiptak-Kechris-corrected for regional correlation structure, and the multiple-testing-corrected Šidák $P$-value. The latter corrects the combined $P$ for $n_{a} / n_{r}$ tests, where $n_{\mathrm{a}}$ is the total number of probes tested in the initial epigenome wide association scan and $n_{r}$ the number of probes in the given region. The Bioconductor package bumphunter ${ }^{31}$ was used to confirm DMRs identified by comb- $p$ with an alternative method. We report the empirical $P$-value, calculated using 1000 permutations. Genes were assigned to probes using the Genomic Regions Enrichment of Annotations Tool (GREAT) package from the Bejerano Lab at Stanford University (http:// bejerano.stanford.edu/great/public/html), ${ }^{32}$ taking into account the functional significance of cis-regulatory regions.

\section{Bisulfite-pyrosequencing}

To technically validate the $450 \mathrm{~K}$ array data at the CYP2E1 DMR, a bisulfitepyrosequencing assay spanning three CpG sites (cg14250048, cg00436603 and cg01465364) was designed using the PyroMark Assay design software (Qiagen, Hilden, Germany). Bisulfite-PCR amplification was performed in duplicate on samples with sufficient remaining DNA using the primers in Supplementary Table 3 and a PCR annealing temperature of $55^{\circ} \mathrm{C}$. DNA methylation was quantified in a subset of 36 samples with sufficient remaining DNA using the Pyromark Q24 system (Qiagen), following the manufacturer's standard instructions, and the Pyro Q24 CpG 2.0.6 software.

\section{RESULTS}

Sociocognitive consequences of exposure

Phenotypic analyses on the selected subsample of the ERA cohort used in this study confirmed previously reported negative associations between exposure to severe early-life institutional deprivation and performance in sociocognitive tests (Figure 1). Romanian adoptees exposed to $>6$ months of deprivation scored significantly lower on tests of both IQ $(P=0.004)$ and ToM $\left(P=3.07 \times 10^{-4}\right)$.

Deprivation-associated DNA methylation differences

We first assessed DNA methylation differences at specific $450 \mathrm{~K}$ array probes between Romanian adoptees categorized as having experienced 'limited' ( $<6$ months in institutional deprivation, $n=17)$ and 'extended' periods of institutional deprivation ( $>6$ months in institutional deprivation, $n=16$; see Table 1 and Supplementary Figure 2 for the top-ranked differentially methylated positions). No probe-wise differences remained significant after correction for multiple correction, although this is not surprising, given the small number of samples available for this study. DNA methylation differences for the 100 top-ranked exposure-associated differentially methylated positions (Supplementary Table 4) were highly correlated with effect sizes at the same loci in a quantitative analysis of exposure duration $\left(r=0.93, P=3.03 \times 10^{-44}\right.$; Supplementary Figure 3$)$, indicating that the effects of severe deprivation at these loci are likely to be cumulative.

We next used comb- $p^{30}$ to identify spatially correlated regions of differential DNA methylation, identifying a significant DMR on chromosome 10 spanning nine sequential $450 \mathrm{~K}$ array probes, which were consistently increased in DNA methylation in the severe early institutional deprivation group (combined $P=2.21 \times 10^{-10}$, corrected Šidák $P=2.98 \times 10^{-5}$ ). This region was also identified using an alternative DMR analysis method (bumphunter ${ }^{31}$ ) as showing significantly elevated DNA methylation in the severely exposed group (adjusted $P=0.002$; Figure 2, Supplementary Figure 4 and Table 2). By comparing the two Romanian adoptee groups with the matched group of children born and adopted within the UK, we were able to show that increased DNA methylation across this DMR is specific to the group that experienced extended deprivation; the control group of UK adoptees was indistinguishable from the Romanian group adopted before the age of 6 months at each of the nine CpG sites comprising the DMR (Figure 2 and Supplementary Figure 4). This $\sim 600$ bp DMR spans the transcription start site and first exon of the cytochrome P450 gene, CYP2E1. There was a significant correlation between DNA methylation levels independently derived from the $450 \mathrm{~K}$ array and bisulfite-pyrosequencing experiments ( $r=0.52, P=0.001$, Supplementary Figure 5$)$.

Association between DNA methylation and deprivation-related sociocognitive and intellectual impairments

We next tested whether exposure-associated DNA methylation differences were associated with established deprivation-related impairments in cognition and deficits in ToM across samples for

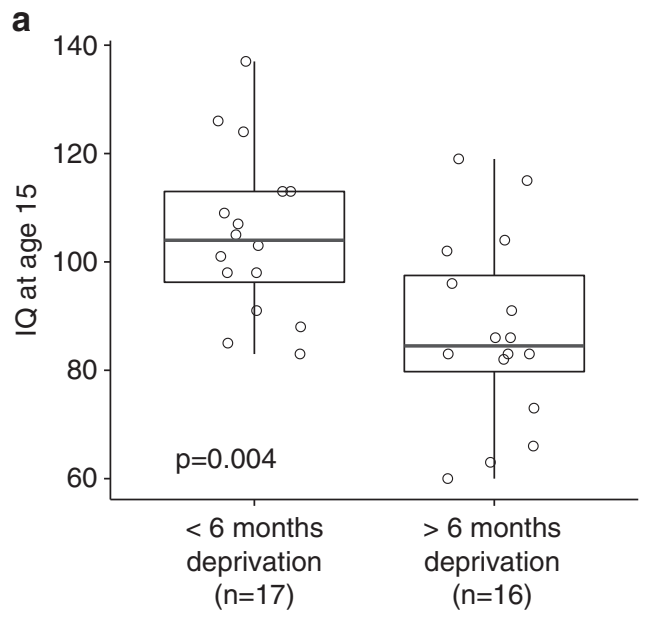

Exposure group b

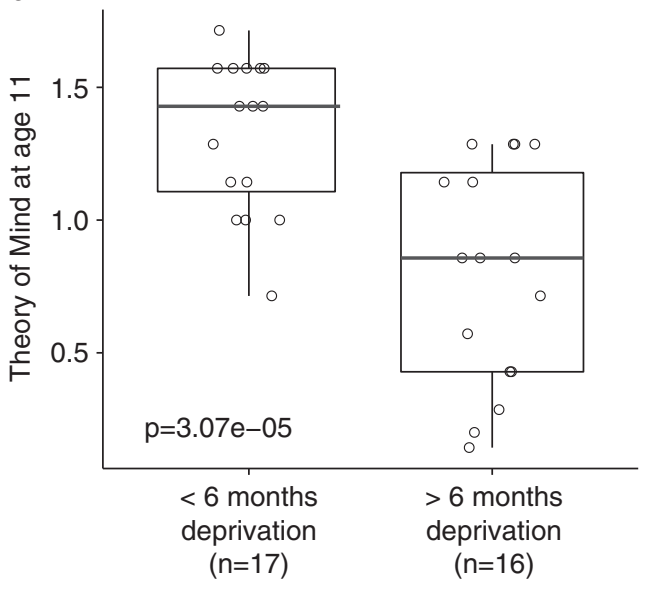

Exposure group

Figure 1. Exposure to severe early-life deprivation is negatively associated with performance in sociocognitive tasks in the English and Romanian Adoptees study (ERA) subsample included in methylomic profiling. Prolonged exposure ( $\geqslant 6$ months) was significantly associated with lower scores for (a) intelligence quotient (IQ) at the age of $15(P=0.004)(\mathbf{b})$ and the Theory of Mind at the age of $11\left(P=3.07 \times 10^{-4}\right)$. 
Table 1. The top-ranked differentially methylated positions associated with exposure to severe institutional deprivation

\begin{tabular}{llccc}
\hline Probe & GREAT gene annotation & P-value & DNA methylation difference & $>6-$ Month exposure (mean) \\
\hline cg11634248 & CHKA, SUV420H1 & $2.35 \times 10^{-5}$ & 0.03 & 0.88 \\
cg14272935 & FGF5 & $6.89 \times 10^{-5}$ & 0.05 & 0.37 \\
cg16668903 & SNX24, PPIC & $9.75 \times 10^{-5}$ & 0.05 \\
cg06969206 & HHIPL1 & $1.08 \times 10^{-4}$ & 0.07 & 0.79 \\
cg22982014 & LGALS4, HNRNPL & $1.26 \times 10^{-4}$ & 0.05 & 0.24 \\
cg24843511 & S100A2, S100A16 & $1.38 \times 10^{-4}$ & 0.03 & 0.24 \\
cg18015809 & GPR110, TNFRSF21 & $1.60 \times 10^{-4}$ & 0.03 & 0.82 \\
cg08157194 & SLC25A17, MCHR1 & $1.63 \times 10^{-4}$ & 0.03 & 0.90 \\
cg04213775 & SLC12A7, NKD2 & $1.68 \times 10^{-4}$ & 0.02 & 0.19 \\
cg07085824 & SGK196 & $1.72 \times 10^{-4}$ & 0.03 & 0.79 \\
\hline
\end{tabular}

Abbreviation: GREAT, Genomic Regions Enrichment of Annotations Tool.

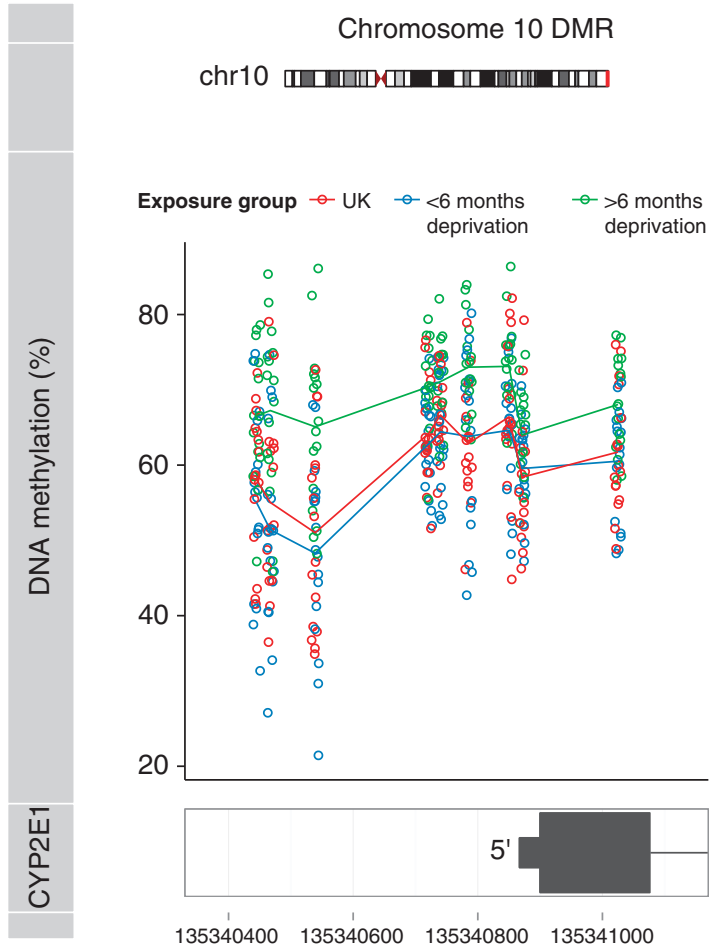

Figure 2. A differentially methylated region (DMR) spanning the transcription start site of CYP2E1 shows significantly increased DNA methylation levels in adoptees exposed to severe early-life adversity. A DMR on chromosome 10 spanning nine sequential 450K array probes (chr10:135340445-135341026) was identified by comb-p. DNA methylation across this region is significantly elevated (combined $P=2.21 \times 10^{-10}$; corrected Šidák $P=2.98 \times 10^{-5}$ ) in individuals exposed to severe long-term ( $\geqslant 6$ months) institutional deprivation compared with the low-exposure ( $<6$ months) group and UK control group. Data for an extended region around this DMR are shown in Supplementary Figure 4.

which $450 \mathrm{~K}$ array data were available. For the 100 top-ranked exposure-group differentially methylated positions, there was a highly significant negative correlation between exposure-group DNA methylation differences and effect sizes at the same probes for both IQ $\left(r=-0.82, P=4.48 \times 10^{-25}\right.$, Supplementary Figure 6) and ToM $\left(r=-0.89, P=2.23 \times 10^{-35}\right.$, Figure 3a). Furthermore, using comb-p to identify DMRs for sociocognitive and intellectual impairments, we found that DNA methylation across the nine CpG sites in the deprivation-associated CYP2E1 DMR on chromosome
10 was significantly associated with ToM (combined $P=4.87 \times 10^{-9}$; Table 3 and Figure $3 \mathrm{~b}$ ) and cognitive impairment (combined $P=2.912 \times 10^{-5}$ ).

\section{DISCUSSION}

Using samples from a unique 'natural experiment' following children exposed to prolonged severe institutional deprivation, we provide evidence for significant alterations in DNA methylation in response to severe early-life social adversity in humans. We identified a DMR that was associated with extended institutional deprivation across nine adjacent CPG sites spanning the transcription start site and first exon of the cytochrome P450 gene CYP2E1. Elevated DNA methylation across this DMR was specific to the group exposed to more than 6 months in Romanian institutions; early-adopted Romanian adoptees were indistinguishable from the control group of UK adoptees-an effect that mirrors prior findings relating deprivation and psychiatric disorders and cognition. ${ }^{13}$ DNA methylation across the nine CpG sites in the CYP2E1 DMR was also associated with ToM performance and cognitive impairment.

The CYP2E1 protein is a member of the cytochrome P450 (CYPs) super family of enzymes, with a role in the metabolism of various exogenous compounds including drugs of abuse and neurotoxins. ${ }^{33}$ It is also involved in gluconeogenesis and the synthesis of cholesterol, steroids and other lipids. ${ }^{34}$ CYP2E1 is most abundantly expressed in the liver, although like other CYPs it is present and is active in the human brain, including the frontal cortex, hippocampus, amygdala, hypothalamus and cerebellum (GTEx Analysis Release V4: dbGaP Accession phs000424.v4.p1 (ref. 33)). There is evidence to suggest that CYPs in the brain may have a role in modulating behavior ${ }^{35}$ and cognitive processes (for example, shown by imaging genetic studies ${ }^{36}$ ) as well as susceptibility to central nervous system diseases and drug dependence. $^{37}$

It is currently unknown which molecular pathways in the brain might be affected by changes in CYP2E1 function, and how deprivation-related sociocognitive deficits might be mechanistically connected to epigenetic variation regulating CYP2E1. Of note, increased methylation of a CpG site in close proximity $(<1 \mathrm{~kb})$ to our DMR in neonates has been recently associated with prenatal exposure to selective serotonin reuptake inhibitors ${ }^{38}$ (Supplementary Figure 4). Although this specific CpG site was not within the DMR identified in our study, it was nominally significantly associated with exposure to adversity $(P=0.034)$. Prenatal selective serotonin reuptake inhibitor exposure, similar to severe early-life adversity, has been implicated as a risk factor for long-term cognitive deficits and psychopathology. ${ }^{39}$ In a mouse model, it was shown that chronic psychoemotional stress reduced CYP2E1 protein levels by one half, suggesting that stress exposure 
Table 2. Association statistics for the nine individual probes in the chromosome $10 \mathrm{DMR}$

\begin{tabular}{|c|c|c|c|c|c|c|c|}
\hline Probe & Position & $\begin{array}{l}\mathrm{P}>6 \text {-month-versus } \\
<6 \text {-month exposure }\end{array}$ & $\begin{array}{l}\text { P UK versus } \\
<6 \text {-month } \\
\text { exposure }\end{array}$ & $\begin{array}{c}\text { DNA methylation difference } \\
>6 \text {-month- versus } \\
<6 \text {-month exposure }\end{array}$ & $\begin{array}{c}>6-\text { Month } \\
\text { exposure (mean) }\end{array}$ & $\begin{array}{c}<6-\text { Month } \\
\text { exposure (mean) }\end{array}$ & UK (mean) \\
\hline $\operatorname{cg} 07381788$ & 135340445 & 0.003 & 0.419 & 0.12 & 0.67 & 0.55 & 0.58 \\
\hline cg10986462 & 135340539 & $3.00 \times 10^{-4}$ & 0.545 & 0.17 & 0.65 & 0.48 & 0.51 \\
\hline cg01465364 & 135340721 & 0.001 & 0.554 & 0.08 & 0.70 & 0.63 & 0.64 \\
\hline cg00436603 & 135340740 & 0.007 & 0.316 & 0.07 & 0.71 & 0.64 & 0.67 \\
\hline cg19837601 & 135340871 & 0.053 & 0.666 & 0.04 & 0.64 & 0.60 & 0.58 \\
\hline $\operatorname{cg} 21024264$ & 135341025 & 0.004 & 0.660 & 0.08 & 0.68 & 0.61 & 0.62 \\
\hline
\end{tabular}

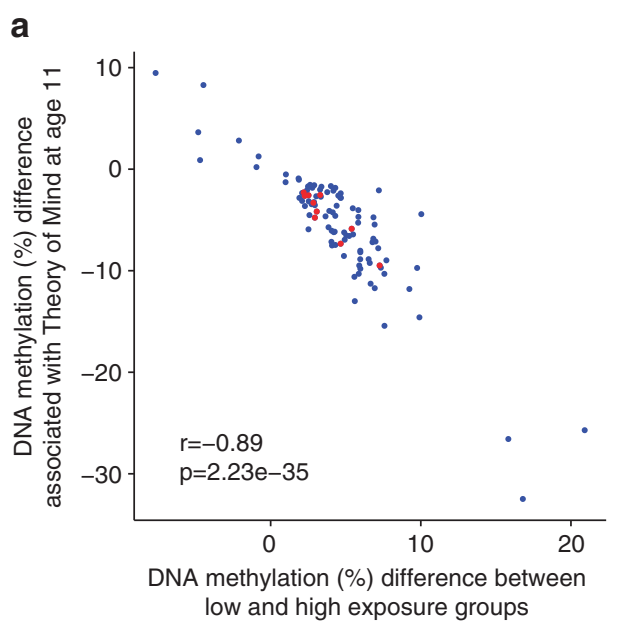

low and high exposure groups
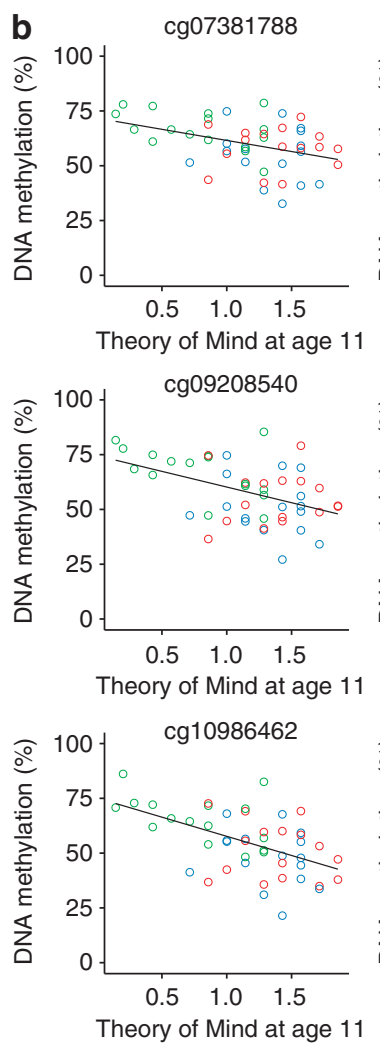
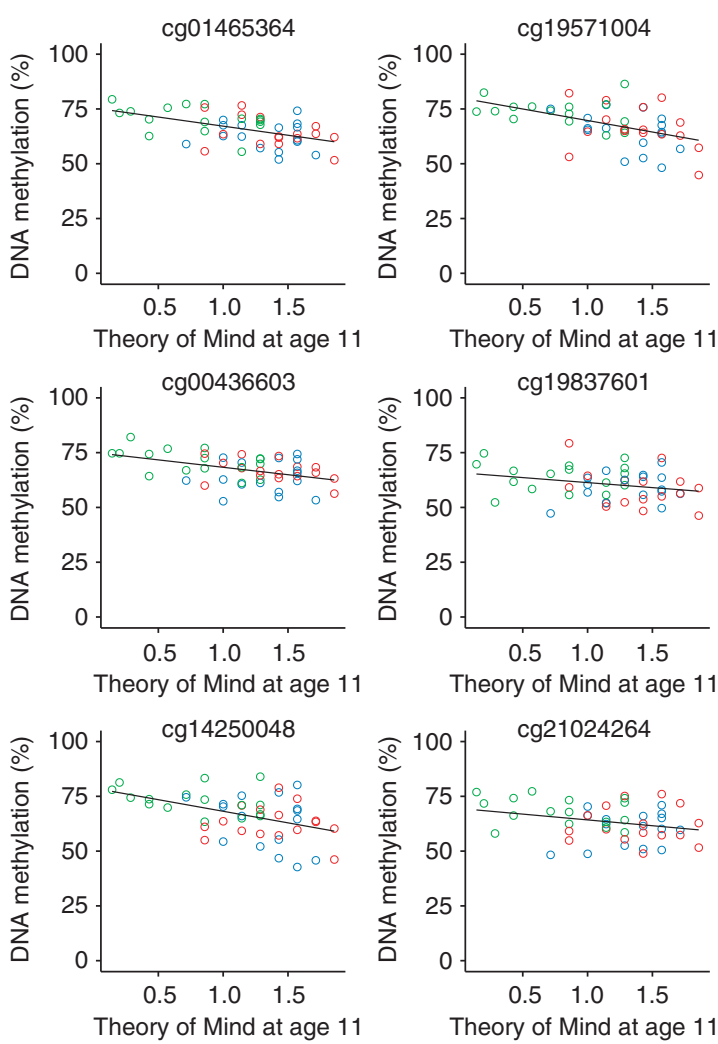

Figure 3. Exposure-associated DNA methylation variation is associated with Theory of Mind test performance. (a) For the 100 top-ranked exposure-associated differentially methylated positions (DMPs; see Supplementary Table 4), effect sizes for association with exposure group correlated significantly with effect sizes for association with Theory of Mind at the age of $11\left(r=-0.89, P=2.23 \times 10^{-35}\right)$. The top 10 exposureassociated DMPs (see Table 1) are highlighted in red. (b) The CYP2E1 DMR associated with exposure group is also significantly associated with the Theory of Mind (combined $P=4.87 \times 10^{-9}$, corrected Šidák $P=8.79 \times 10^{-5}$ ). Associations between DNA methylation and Theory of Mind are shown for the nine probes constituting the DMR, with the previously used coloring scheme (UK adoptees: red, short deprivation exposure group: blue, extended exposure group: green).

might have a role in CYP2E1 regulation. ${ }^{40}$ Our observation that DNA methylation differences in the regulatory region of CYP2E1 associated with extended deprivation also correlated with reduced IQ, and ToM is consistent with the view that shared processes may be involved in mediating the observed deficits in both social and intellectual functioning. ${ }^{17,41}$

There is now a large body of evidence and a strong, scientific consensus that childhood stress and early adversity, especially in such extreme forms as the institutional deprivation experienced by the ERA sample, are associated with disturbances of childhood mental health and life-long risks of chronic disorders of mental and physical health. ${ }^{42}$ The exact mechanisms by which signals from the social environment impinge on the developing brain to shape the neural circuitry and what role epigenetic processes may have in stabilizing developmental trajectories across the lifespan are only just beginning to be elucidated. Brain structure and function are especially responsive to experience early in life, and development is characterized by the key developmental stages of heightened plasticity. ${ }^{43}$ Recent research shows that during these critical periods the genome may be particularly vulnerable to 
Table 3. Association statistics of the nine probes in the chromosome 10 DMR with Theory of Mind

\begin{tabular}{lclc}
\hline Probe & Position & P-value & Effect size $^{\mathrm{a}}$ \\
\hline cg07381788 & 135340445 & 0.006 & -0.19 \\
cg09208540 & 135340467 & 0.001 & -0.27 \\
cg10986462 & 135340539 & $1.10 \times 10^{-4}$ & -0.32 \\
cg01465364 & 135340721 & $2.78 \times 10^{-4}$ & -0.15 \\
cg00436603 & 135340740 & 0.002 & -0.13 \\
cg14250048 & 135340785 & 0.001 & -0.20 \\
cg19571004 & 135340850 & $3.12 \times 10^{-4}$ & -0.20 \\
cg19837601 & 135340871 & 0.072 & -0.08 \\
cg21024264 & 135341025 & 0.052 & -0.10 \\
\hline
\end{tabular}

${ }^{a}$ Abbreviations: DMR, differentially methylated region. Effect size is scaled to the maximum Theory of Mind score.

epigenetic disruption. ${ }^{44}$ With regard to the ERA sample, it can be speculated that the lack of emotional, sensory and cognitive stimulation associated with deprivation of personalized care during sensitive periods in infant life might have led to epigenetic changes resulting in insufficient fine-tuning of the brain circuitry mediating socioemotional behaviors and underlying higher cognitive function.

One previous study has investigated epigenetic alterations in 8year-old institutionalized children. Differential methylation patterns (at an extremely non-stringent uncorrected $P<0.01$ ) were found at $914 \mathrm{CpG}$ sites, with $\sim 90 \%$ of these nominally differentially methylated sites showing elevated methylation in the institutionalized group. ${ }^{45}$ In addition to the analytical differences between the studies, samples in this prior study were not exposed to significant deprivation, which may explain the difference in number and magnitude of exposure-associated changes.

Our study has a number of important limitations. First, the number of samples profiled in this study is small, and replication in cohorts with similar types of deprivation experience is warranted. However, the ERA represents a unique natural experiment cohort, and access to equivalent samples exposed to a similar level of adversity for replication is by necessity difficult. Second, the small number of samples means that it is underpowered to formally assess whether deprivation effects on sociocognitive processes are mediated by epigenetic effects. Furthermore, our analyses were cross-sectional, and it is not possible to causally link exposure to the variation we observe. It cannot be ruled out that the observed differences in DNA methylation were caused by deprivation-related impairments observed in the high-risk group, or by other confounding factors such as adverse prenatal conditions (although no differences in birth weight were observed between the long- and short-exposed Romanian adoptees) or medication use. Finally, the observed differences in DNA methylation were observed in buccal cells, and the extent to which peripheral markers index epigenetic variation in central nervous tissue is still debated. ${ }^{12,46}$ of note, buccal cells derive from the same embryonic cells as brain tissue (ectoderm) and have less cellular heterogeneity compared with whole blood. ${ }^{47}$ Although there are well-documented tissue-specific differences in DNA methylation, exposure-associated changes in DNA methylation can be identified in many cell types, and peripheral tissues may have some utilities as potential biomarkers of exposure or disease. ${ }^{12,46,48}$ Despite these limitations, our data are consistent with the notion that environmental insults of sufficient biological impact during early development might be associated with epigenetic variation detectable in peripheral cells, and provide further support for a role of epigenetic processes in linking the effects of early-life adversity to cognitive and neurobiological phenotypes.
To conclude, children exposed to extreme early institutional deprivation were characterized by significantly increased DNA methylation across a region of the CYP2E1 gene with putative functional significance for brain function. These findings support the notion that severe social adversity may induce epigenetic variation in human subjects. Future studies should replicate and extend this finding with larger samples and investigate longitudinal changes in DNA methylation over time as a function of post-adversity environments and genomic variations, and relate these to changes in phenotype. It will be important to further investigate the neurobiological significance of these changes by linking DMRs to brain structure and function.

\section{CONFLICT OF INTEREST}

EJS Sonuga-Barke obtained speaker fees, consultancy, research funding and conference support from Shire Pharma. He also received Speaker fees from Janssen Cilag and Consultancy from Neurotech solutions, Aarhus University, Copenhagen University and Berhanderling, Skolerne, Copenhagen, KU Leuven. He obtained Book royalties from OUP and Jessica Kingsley. He also received grants from MRC, ESRC, Wellcome Trust, Solent NHS Trust, European Union, Child Health Research Foundation New Zealand, NIHR, Nuffield Foundation, Fonds Wetenschappelijk Onderzoek-Vlaanderen (FWO) and MQ - Transforming Mental Health. The remaining authors declare no conflicts of interest.

\section{ACKNOWLEDGMENTS}

The current ERA study is supported by ESRC grant ES/I037970/1 and MRC grant MR/ K022474/1 to EJSS-B. RK acknowledges grant support from the Deutsche Forschungsgemeinschaft (DFG, KU 2479/3-1, KU 2479/3-2). SJM is funded by the EU-FP7 Marie Curie ITN EpiTrain (REA grant agreement no. 316758).

\section{REFERENCES}

1 Davidson RJ, McEwen BS. Social influences on neuroplasticity: stress and interventions to promote well-being. Nat Neurosci 2012; 15: 689-695.

2 Repetti RL, Taylor SE, Seeman TE. Risky families: family social environments and the mental and physical health of offspring. Psychol Bull 2002; 128: 330-366.

3 Nelson CA 3rd, Bos K, Gunnar MR, Sonuga-Barke EJ. The neurobiological Toll of Early Human Deprivation. Monogr Soc Res Child Dev 2011; 76: 127-146.

4 Turecki G, Meaney M. Effects of the social environment and stress on glucocorticoid receptor gene methylation: a systematic review. Biol Psychiatry 2016; 79: 87-96, in press.

5 Zhang TY, Meaney MJ. Epigenetics and the environmental regulation of the genome and its function. Annu Rev Psychol 2010; 61: 439-466.

6 Heijmans BT, Tobi EW, Stein AD, Putter H, Blauw GJ, Susser ES et al. Persistent epigenetic differences associated with prenatal exposure to famine in humans. Proc Natl Acad Sci USA 2008; 105: 17046-17049.

7 Essex MJ, Boyce WT, Hertzman C, Lam LL, Armstrong JM, Neumann SM et al. Epigenetic vestiges of early developmental adversity: childhood stress exposure and DNA methylation in adolescence. Child Dev 2013; 84: 58-75.

8 Borghol N, Suderman M, McArdle W, Racine A, Hallett M, Pembrey M et al. Associations with early-life socio-economic position in adult DNA methylation. Int $J$ Epidemiol 2012; 41: 62-74.

9 McGowan PO, Sasaki A, D'Alessio AC, Dymov S, Labonte B, Szyf M et al. Epigenetic regulation of the glucocorticoid receptor in human brain associates with childhood abuse. Nat Neurosci 2009; 12: 342-348.

10 Labonte B, Suderman M, Maussion G, Navaro L, Yerko V, Mahar I et al. Genomewide epigenetic regulation by early-life trauma. Arch Gen Psychiatry 2012; 69: 722-731.

11 Labonte B, Yerko V, Gross J, Mechawar N, Meaney MJ, Szyf M et al. Differential glucocorticoid receptor exon $1(\mathrm{~B}), 1(\mathrm{C})$, and $1(\mathrm{H})$ expression and methylation in suicide completers with a history of childhood abuse. Biol Psychiatry 2012; 72: 41-48.

12 Mill J, Heijmans BT. From promises to practical strategies in epigenetic epidemiology. Nat Rev Genet 2013; 14: 585-594.

13 Rutter M, Sonuga-Barke EJ, Beckett C, Castle J, Kreppner J, Kumsta R et al. Deprivation-specific psychological patterns: effects of institutional deprivation. Monogr Soc Res Child Dev 2010; 75: 232-247.

14 Kumsta R, Kreppner J, Kennedy M, Knights N, Rutter M, Sonuga-Barke E. Psychological consequences of early global deprivation. Eur Psychol 2015; 20: 138-151. 
15 Kreppner J, Kumsta R, Rutter M, Beckett C, Castle J, Stevens S et al. Developmental course of deprivation-specific psychological patterns: early manifestations, persistence to age 15, and clinical features. Monogr Soc Res Child Dev 2010; 75: 79-101.

16 Kumsta R, Kreppner J, Rutter M, Beckett C, Castle J, Stevens S et al. III. Deprivationspecific psychological patterns. Monogr Soc Res Child Dev 2010; 75: 48-78.

17 Colvert E, Rutter M, Kreppner J, Beckett C, Castle J, Groothues C et al. Do Theory of Mind and executive function deficits underlie the adverse outcomes associated with profound early deprivation? Findings from the English and Romanian adoptees study. J Abnorm Child Psychol 2008; 36: 1057-1068.

18 Association AP. Diagnostic and Statistical Manual of Mental Disorders: DSM-5. 5th edn. American Psychiatric Publishing: Arlington, VA, USA, 2013.

19 Zeanah CH, Smyke AT, Koga SF, Carlson E. Attachment in institutionalized and community children in Romania. Child Dev 2005; 76: 1015-1028.

20 Stevens SE, Sonuga-Barke EJS, Kreppner JM, Beckett C, Castle J, Colvert E et al. Inattention/overactivity following early severe institutional deprivation: presentation and associations in early adolescence. J Abnorm Child Psychol 2008; 36: 385-398.

21 Freeman B, Smith N, Curtis C, Huckett L, Mill J, Craig IW. DNA from buccal swabs recruited by mail: evaluation of storage effects on long-term stability and suitability for multiplex polymerase chain reaction genotyping. Behav Genet 2003; 33: 67-72.

22 Team RCR. A Language and Environment for Statistical Computing. R Foundation for Statistical Computing: Vienna, Austria, 2014.

23 Davis S, Du P, Bilke S, Triche TJ, Bootwalla M. Methylumi: Handle Illumina methylation data. R Package Version 2140, 2015.

24 Aryee MJ, Jaffe AE, Corrada-Bravo H, Ladd-Acosta C, Feinberg AP, Hansen KD et al. Minfi: a flexible and comprehensive Bioconductor package for the analysis of Infinium DNA methylation microarrays. Bioinformatics 2014; 30: 1363-1369.

25 Pidsley R, Wong CCY, Volta M, Lunnon K, Mill J, Schalkwyk LC. A data-driven approach to preprocessing Illumina $450 \mathrm{~K}$ methylation array data. BMC Genomics 2013; 14: 293.

26 Chen YA, Lemire M, Choufani S, Butcher DT, Grafodatskaya D, Zanke BW et al. Discovery of cross-reactive probes and polymorphic CpGs in the Illumina Infinium HumanMethylation450 microarray. Epigenetics 2013; 8: 203-209.

27 Wechsler D. Manual for the Wechsler Intelligence Scale for Children. Psychological Corporation: London, 1992; Vol. 3.

28 Sattler JM. Age effects on Wechsler adult intelligence scale-revised tests. J Consult Clin Psychol 1982; 50: 785-786.

29 Happe FG. An advanced test of theory of mind: understanding of story characters' thoughts and feelings by able autistic, mentally handicapped, and normal children and adults. J Autism Dev Disord 1994; 24: 129-154.

30 Pedersen BS, Schwartz DA, Yang IV, Kechris KJ. Comb-p: software for combining, analyzing, grouping and correcting spatially correlated P-values. Bioinformatics 2012; 28: 2986-2988.

31 Jaffe AE, Murakami $\mathrm{P}$, Lee $\mathrm{H}$, Leek JT, Fallin MD, Feinberg AP et al. Bump hunting to identify differentially methylated regions in epigenetic epidemiology studies. Int J Epidemiol 2012; 41: 200-209.

32 McLean CY, Bristor D, Hiller M, Clarke SL, Schaar BT, Lowe CB et al. GREAT improves functional interpretation of cis-regulatory regions. Nat Biotechnol 2010; 28: $495-501$.
33 Strobel HW, Thompson CM, Antonovic L. Cytochromes P450 in brain: function and significance. Curr Drug Metab 2001; 2: 199-214.

34 Tanaka E, Terada M, Misawa S. Cytochrome P450 2E1: its clinical and toxicological role. J Clin Pharm Ther 2000; 25: 165-175.

35 Penas-Lledo EM, Dorado P, Pacheco R, Gonzalez I, Llerena A. Relation between CYP2D6 genotype, personality, neurocognition and overall psychopathology in healthy volunteers. Pharmacogenomics 2009; 10: 1111-1120.

36 Stingl JC, Esslinger C, Tost H, Bilek E, Kirsch P, Ohmle B et al. Genetic variation in CYP2D6 impacts neural activation during cognitive tasks in humans. Neuroimage 2012; 59: 2818-2823.

37 Ferguson CS, Tyndale RF. Cytochromes P450 in brain: emerging evidence for biological significance. Trends Pharmacol Sci 2011; 32: 708-714.

38 Gurnot C, Martin-Subero I, Mah SM, Weikum W, Goodman SJ, Brain U et al. Prenatal antidepressant exposure associated with CYP2E1 DNA methylation change in neonates. Epigenetics 2015; 10: 1-12.

39 Moses-Kolko EL, Bogen D, Perel J, Bregar A, Uhl K, Levin B et al. Neonatal signs after late in utero exposure to serotonin reuptake inhibitors: literature review and implications for clinical applications. JAMA 2005; 293: 2372-2383.

40 Maksymchuk O, Chashchyn M. The impact of psychogenic stressors on oxidative stress markers and patterns of CYP2E1 expression in mice liver. Pathophysiology 2012; 19: 215-219.

41 Kumsta R, Rutter M, Stevens S, Sonuga-Barke EJ. IX. Risk, causation, mediation, and moderation. Monogr Soc Res Child Dev 2010; 75: 187-211.

42 Shonkoff JP, Boyce WT, McEwen BS. Neuroscience, molecular biology, and the childhood roots of health disparities: building a new framework for health promotion and disease prevention. JAMA 2009; 301: 2252-2259.

43 Fox SE, Levitt $\mathrm{P}$, Nelson CA. How the timing and quality of early experiences influence the development of brain architecture. Child Dev 2010; 81: 28-40.

44 Fagiolini M, Jensen CL, Champagne FA. Epigenetic influences on brain development and plasticity. Curr Opin Neurobiol 2009; 19: 207-212.

45 Naumova OY, Lee $M$, Koposov R, Szyf $M$, Dozier M, Grigorenko EL. Differential patterns of whole-genome DNA methylation in institutionalized children and children raised by their biological parents. Dev Psychopathol 2012; 24: 143-155.

46 Hannon E, Lunnon K, Schalkwyk L, Mill J. Interindividual methylomic variation across blood, cortex, and cerebellum: implications for epigenetic studies of neurological and neuropsychiatric phenotypes. Epigenetics 2015; 10: 1024-1032.

47 Lowe R, Gemma C, Beyan H, Hawa MI, Bazeos A, Leslie RD et al. Buccals are likely to be a more informative surrogate tissue than blood for epigenome-wide association studies. Epigenetics 2013; 8: 445-454.

48 Hou L, Zhang X, Wang D, Baccarelli A. Environmental chemical exposures and human epigenetics. Int J Epidemiol 2012; 41: 79-105.

This work is licensed under a Creative Commons Attribution 4.0 nternational License. The images or other third party material in this article are included in the article's Creative Commons license, unless indicated otherwise in the credit line; if the material is not included under the Creative Commons license, users will need to obtain permission from the license holder to reproduce the material. To view a copy of this license, visit http://creativecommons.org/licenses/ by/4.0/
}

Supplementary Information accompanies the paper on the Translational Psychiatry website (http://www.nature.com/tp) 